\title{
Clinical trials of antiarrhythmic therapies and optimizing health care resource deployment: the need for a paradigm shift
}

\author{
Sanjeev Saksena
}

Published online: 13 January 2012

(C) Springer Science+Business Media, LLC 2012

Randomized clinical trials (RCTs) of antiarrhythmic therapies have proliferated since they replaced observational clinical reports over two decades ago. Challenged initially at almost every step, from ethics to endpoints, RCTs have formed the bedrock of evidence-based medicine and practice guidelines in cardiac arrhythmias. Yet the implementation of evidence-based diagnostic or therapeutic guidelines suitable for clinical practice has not been without difficulty. Some have evoked an uneasy acceptance in many quarters and others even more difficult execution in medical practice. Examples abound. The rate versus rhythm debate in atrial fibrillation (AF) management is a prominent one. Clinical trial outcomes were more readily accepted by generalists than specialists, with the latter perplexed by the unexpected outcome that ran counter to physiologic principles and lacked a robust and complete explanation [1,2]. Prophylaxis of sudden death events in clinical practice using implantable defibrillator devices in high-risk patients resulted in unused device therapy in a very large proportion of all recipients [3]. These non-uniform outcomes are also seen in cardiac resynchronization therapy and ablation of an increasingly broad spectrum of the AF population $[4,5]$.

Recently, these foundational clinical trials have been revisited in part or in great measure [6,7]. These reports have been both enlightening and concerning. They enlighten us by revealing common underlying threads of behavior in arrhythmia disorders. They evoke concern in two areas.

S. Saksena $(\bowtie)$

UMDNJ-Robert Wood Johnson School of Medicine,

161 Washington Valley Road, Suite 201,

Warren, NJ 07059, USA

e-mail: DRSSEPNJ@aol.com

S. Saksena

e-mail: CMENJ@aol.com
Firstly, by revealing serious adverse effects of long established and widely employed therapeutic interventions; and secondly, that our current paradigms of clinical trial design and patient enrollment result in highly variable benefits with a serious proportion of patients receiving little or no longterm benefit from the intervention. Statisticians remind us regularly of the perils and pitfalls of both retrospective visits to clinical trial data and subgroup analyses. To paraphrase a distinguished editor of this Journal, there should be due caution around "relying on subgroup analyses or declaring a difference when there is none and vice versa." This concern arises mostly due to lack of statistical power in the original trial design for the subgroup analysis, which may actually mask potential benefit or harm. At best, we accept the premise that these subgroup analyses can be construed as hypothesis generating. We do appear to be better at selecting subgroups that achieve significant harm in clinical trials with safety monitoring.

However, the implications of looking away from highly visible patterns of behavior in clinical outcomes have enormous implications for both patients subjected to unnecessary care and wasted resources in a resource-limited global health care environment. For example, with direct and indirect cardiac resynchronization defibrillator expenditures exceeding three billion US dollars in this year past, nonresponders to this therapy contributed over one billion dollars in cost. Some of these individuals could be simply identified by baseline characteristics at implant, according to trial subgroup analyses. In this authors' view, it is time for a paradigm shift in health care resource deployment that does take into account important subgroup data.

What are common threads of behavior in arrhythmia outcomes? They appear to lie in three different areas. Firstly, a clear understanding of important baseline variables in recruited populations that will impact outcomes. Managing 
these variables is not simply a matter of balanced distribution required to test the trial hypothesis. Arrhythmia patients have important comorbidities that affect outcomes. In a recent report on the Atrial Fibrillation Follow-up Investigation of Rhythm Management (AFFIRM) trial, we noted a general effect of well-defined baseline variables, such as coronary or pulmonary disease and heart failure, that limited beneficial outcomes with all interventions tested [6]. Similar situations exist in trials employing implantable cardioverterdefibrillators (ICDs) for sudden death prophylaxis in patients with renal dysfunction and class IV heart failure $[4,8]$. Other baseline variables influence outcomes only with specific interventions, for example, amiodarone use in atrial fibrillation patients with preexisting or treated thyroid disease or high energy shocks in patients with impaired left ventricular function and comorbidities [6, 7].

Secondly, temporal aspects of disease progression at the onset or during the tenure of the clinical trial have outcome consequences. Arrhythmia status and disease state progression is a poorly defined relationship for both atrial and ventricular arrhythmias. It is however critical to longitudinal healthcare delivery and cost. It can also result in trial imbalances that are not obvious. Arrhythmia progression is now measurable. For example, atrial fibrillation (AF) burden can be highly variable in patients, even when only paroxysmal or persistent AF patients are recruited based on presence of structural heart disease or the duration of the persistent AF $[9,10]$. Progression of AF burden in persistent AF can be an important time-dependent variable impacting outcomes [10]. This is now indirectly recognized in a new ESC classification of persistent AF [11]. In the AFFIRM trial, progression of heart failure and ischemia impaired outcomes [6]. Survival benefits may also vary by remoteness from other beneficial interventions such as revascularization therapy [12]. Inadequate control of hyperlipidemia implicit in limited statin use or atrial fibrillation emergence has also reduced benefits of ICD therapy. In fact, geographic variability in ICD benefits seen in the SCD-HeFT trial could relate to such factors.

Lastly, individual antiarrhythmic therapies have an independent impact on outcomes, only in part related to proarrhythmia. High-energy shocks may cause ventricular stunning and cardiac enzyme release which has variable tolerance in patients with left ventricular dysfunction [7]. Amiodarone promotes more serious cardiovascular hospitalizations and increases noncardiac mortality by hitherto unrecognized mechanisms [6]. To offset these deleterious trends, arrhythmia suppression or reversion achieved by the antiarrhythmic therapies may improve outcomes. In AFFIRM, a high degree of rhythm control was associated with improved outcomes. Principal trial outcomes reflect the interplay of all these forces.

All in all, these reports identify issues to be addressed by both clinical trial designs and clinical practice guidelines. In clinical trials, the observed primary outcome is a composite outcome of constituent clinical subgroups with variable benefit, and in some cases, deleterious effects from arrhythmia therapy. Arrhythmias live in the disease substrate that generates them and the parent disease must be weighed for outcomes impact. Major comorbidity management strategies need to be monitored and addressed in the tenure of clinical trials. Prevention of progression of the native disease and the arrhythmia should be goals in therapy with aggressive upstream therapy [13]. The impact of antiarrhythmic therapies in prevention of arrhythmia progression, and secondarily in disease progression, can also have an important positive impact, but these are often inadequately measured. To achieve this result, the extent of arrhythmia control needs to be accurately quantified for potential beneficial thresholds. Finally, to select subgroups that achieve the majority of the observed benefit in trials is trickier. Careful selection of at least a few scientifically and practically relevant subgroups is challenging but needed. This is where prospective risk stratification has one of its greatest contributions [14]. In trials with serious impact on clinical practice and health care delivery, failure to address risk stratifiers is indeed a major flaw in trial design. Analysis of similar subgroups across multiple trials and consistency in their behavior should be performed and monitored by practice guidelines. Evolving living guidelines become paramount to avoid wasteful expenditures of resources.

New considerations should arise in developing future clinical trials. Firstly and most importantly, the underlying mechanisms of the arrhythmia as well as the underlying disease progression should be well studied in humans prior to empiric investment in premature and profoundly expensive clinical therapies used in trials. Anatomically based arrhythmia interventions have invariably spurred the need for a greater understanding of human arrhythmia mechanisms. First seen in surgical arrhythmia ablation, this is recently best exemplified by the increasing need for mapping and proliferation of extrapulmonary sites of ablation in atrial fibrillation.

Second, defining the elemental constituents of the observed trial outcomes bring with it clear identification of patient populations that derive reliable benefit and suffer reproducible harm from the therapy. As an example, prospective risk stratification strategies, notably absent from some prominent ICD trials, can be used to cull out highrisk populations to achieve these goals as well. Careful consideration of benefit and harm of antiarrhythmic therapies is the third important element. When there are choices in therapy delivery, for example between electrical therapies for tachycardias, a reasonable hypothesis would examine potential benefit of the choices in the experiment rather than empirical intervention selection.

The need for a paradigm shift in clinical trials and guideline formulation leading to better focused evidence-based 
arrhythmia care has never been more obvious than at this time. Better deployment of antiarrhythmic therapy is essential to convince patients and practitioners of its value and for robust allocation of available budgetary resources by health care delivery systems. In a time of competition for healthcare resource allocation, arrhythmia populations that derive the majority of the observed benefit need to be supported and those that experience poor outcomes eliminated before guidelines are released. An ongoing process to monitor new data and modify guidelines on the basis of aggregated analysis needs to be solidified. In a resource-limited global health care delivery environment, reliable progress in antiarrhythmic therapy is best achieved with critical review of the evidence.

A final word of reflection. 2011 was another successful year for the Journal. This was only achieved by the hard work and unstinting support of our editors, editorial board, reviewers, and worldwide editorial staff. We especially recognize the importance of our indefatigable reviewers in this issue. The international commitment of the Journal is reflected in its global growth and outreach to all those who care for arrhythmia patients worldwide. We look forward to continuing this direction in the coming year.

\section{Sanjeev Saksena MD \\ Editor in Chief}

\section{References}

1. De Denus, S., Sanoski, C. A., Carlsson, J., Opolski, G., \& Spinler, S. A. (2005). Rate vs. rhythm control in patients with atrial fibrillation: a meta-analysis. Archives of Internal Medicine, 165, 258-262.

2. Corley, S. D., Epstein, A. E., DiMarco, J. P., et al. (2004). Relationships between sinus rhythm, treatment, and survival in the Atrial Fibrillation Follow-Up Investigation of Rhythm Management (AFFIRM) Study. Circulation, 109(12), 1509-1513.

3. Saksena, S. (2011). Twenty-five years of implantable defibrillator practice in the USA: are we ready for globalization? Journal of Interventional Cardiac Electrophysiology, 30(1), 1-3.

4. Goldenberg, I., Hall, W. J., Beck, C. A., et al. (2011). Reduction of the Risk of recurring heart failure events with cardiac resynchronization therapy MADIT-CRT (Multicenter Automatic Defibrillator
Implantation Trial with Cardiac Resynchronization Therapy). Journal of the American College of Cardiology, 58, 729-737.

5. Weerasooriya, R., Khairy, P., Litalien, J., et al. (2011). Catheter ablation for atrial fibrillation: are results maintained at 5 years of follow-up? Journal of the American College of Cardiology, 57(2), 160-166.

6. Saksena, S., Slee, A., Waldo, A. L., et al. (2011). Cardiovascular outcomes in the AFFIRM Trial (Atrial Fibrillation Follow-Up Investigation of Rhythm Management): an assessment of individual antiarrhythmic drug therapies compared with rate control with propensity score-matched analyses. Journal of the American College of Cardiology, 58, 1975-1985.

7. Moss, A. J., Greenberg, H., Case, R. B., et al. (2004). Long-term clinical course of patients after termination of ventricular tachyarrhythmia by an implanted defibrillator. Circulation, 110, 37603765.

8. Cygankiewicz, I., Gillespie, J., Zareba, W., et al. (2009). Predictors of long-term mortality in Multicenter Automatic Defibrillator Implantation Trial II (MADIT II) patients with implantable cardioverterdefibrillators. Heart Rhythm, 6(4), 468-473.

9. Saksena, S., Hettrick, D., Koehler, J., Grammatico, A., \& Padeletti, L. (2007). Progression of paroxysmal atrial fibrillation to persistent atrial fibrillation in patients with bradyarrhythmias. American Heart Journal, 154(5), 884-892.

10. Nagarakanti, R., Saksena, S., Hettrick, D., Koehler, J. L., Grammatico, A., \& Padeletti, L. (2011). Progression of new onset to established persistent atrial fibrillation: an implantable devicebased analysis with implications for clinical classification of persistent atrial fibrillation. Journal of Interventional Cardiac Electrophysiology, 32(1), 7-15.

11. European Heart Rhythm Association, European Association for Cardio-Thoracic Surgery, Camm, A. J., Kirchhof, P., Lip, G. Y., et al. (2010). Guidelines for the management of atrial fibrillation: the Task Force for the management of Atrial Fibrillation of the European Society of Cardiology (ESC). Europace, 12(10), 1360 1420.

12. Goldenberg, I., Moss, A. J., McNitt, S., et al. (2006). Time dependence of defibrillator benefit after coronary revascularization in the Multicenter Automatic Defibrillator Implantation Trial (MADIT)II. Journal of the American College of Cardiology, 47(9), 18111817.

13. Desai, A. S., Lewis, E. F., Li, R., Solomon, S. D., et al. (2011). Rationale and design of the treatment of preserved cardiac function heart failure with an aldosterone antagonist trial: a randomized, controlled study of spironolactone in patients with symptomatic heart failure and preserved ejection fraction. American Heart Journal, 162(6), 966-972.

14. Goldenberg, I., Vyas, A. K., Hall, W. J., et al. (2008). Risk stratification for primary implantation of a cardioverter-defibrillator in patients with ischemic left ventricular dysfunction. Journal of the American College of Cardiology, 51(3), 288-296. 